\title{
Recognition of Azole-Resistant Aspergillosis by Physicians Specializing in Infectious Diseases, United States
}

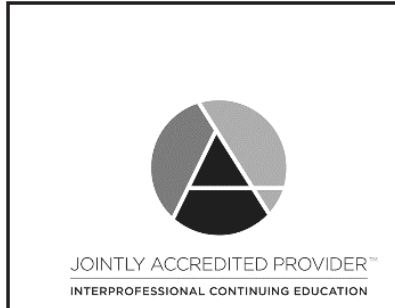

with the extent of their participation in the activity.

All other clinicians completing this activity will be issued a certificate of participation. To participate in this journal CME activity: (1) review the learning objectives and author disclosures; (2) study the education content; (3) take the post-test with a $75 \%$ minimum passing score and complete the evaluation at http://www.medscape.org/journal/eid; and (4) view/print certificate. For CME questions, see page 190.

Release date: December 14, 2017; Expiration date: December 14, 2018

\section{Learning Objectives}

Upon completion of this activity, participants will be able to:

- Assess clinician awareness of and experience with azole-resistant Aspergillus fumigatus infections based on an Emerging Infections Network survey of US infectious disease physicians

- Describe availability and use of $A$. fumigatus susceptibility testing in clinical settings

- Identify regional differences in availability and use of $A$. fumigatus susceptibility testing in clinical settings.

\section{CME Editor}

Jean Michaels Jones, BSN, Technical Writer/Editor, Emerging Infectious Diseases. Disclosure: Jean Michae/s Jones has disclosed no relevant financial relationships.

\section{CME Author}

Laurie Barclay, MD, freelance writer and reviewer, Medscape, LLC. Disclosure: Laurie Barclay, MD, has disclosed the following relevant financial relationships: owns stock, stock options, or bonds from Alnylam; Biogen; Pfizer.

\section{Authors}

Disclosures: Tiffany A. Walker, MD; Shawn R. Lockhart, PhD; Susan E. Beekmann, RN, MPH; Philip M. Polgreen, MD, MPH; Scott Santibanez, MD, MPHTM; Rajal K. Mody, MD, MPH; Karlyn D. Beer, MS, PhD; Tom M. Chiller, MD, MPHTM; and Brendan R. Jackson, MD, MPH, have disclosed no relevant financial relationships.

Tiffany A. Walker, Shawn R. Lockhart,

Susan E. Beekmann, Philip M. Polgreen, Scott Santibanez, Rajal K. Mody, Karlyn D. Beer, Tom M. Chiller, Brendan R. Jackson

Author affiliations: Centers for Disease Control and Prevention, Atlanta, Georgia, USA (T.A. Walker, S.R. Lockhart, S. Santibanez, R.K. Mody, K.D. Beer, T.M. Chiller, B.R. Jackson); University of lowa Carver College of Medicine, lowa City, lowa, USA (S.E. Beekmann, P.M. Polgreen)

DOI: https://doi.org/10.3201/eid2401.170971
Infections caused by pan-azole-resistant Aspergillus fumigatus strains have emerged in Europe and recently in the United States. Physicians specializing in infectious diseases reported observing pan-azole-resistant infections and low rates of susceptibility testing, suggesting the need for wider-scale testing.

Tnfections with strains of Aspergillus fumigatus that are resistant to all azole antifungal medications have become common in western Europe and have been documented in other regions since $1998(1,2)$, but until recently, had 
not been reported in the United States (3). The Infectious Diseases Society of America recommends voriconazole, an azole antifungal medication, as first-line therapy for invasive $A$. fumigatus infections (4). Pan-azole-resistant $A$. fumigatus strains that have $\mathrm{TR}_{34} / \mathrm{L} 98 \mathrm{H}$ and $\mathrm{TR}_{46} / \mathrm{Y} 121 \mathrm{~F} /$ T289A mutations of the Cyp 51A gene have been associated with higher rates of treatment failure and death (2). These mutations may be linked to agricultural and environmental use of azole fungicides (5-7) and not previous azole therapy, because patients with these infections frequently lack prior clinical exposure to azoles (i.e., were azole-naive) (2). These persons were likely exposed to A. fumigatus that developed resistance after exposure to environmental fungicides. Limited genetic diversity between strains isolated from noncontiguous countries suggests a common origin with capacity for extensive geographic spread (1).

Recent limited data suggest a low rate of illness caused by azole-resistant $A$. fumigatus exists in the United States $(1,3,8)$. Isolates with mutations conferring pan-azole resistance have recently been documented (9-11); however, little is known about the broader epidemiology, because there is no national surveillance of Aspergillus spp. In addition, little is known about the degree to which A. fumigatus resistance testing is available to US clinicians. These data could inform future testing and clinical practice.

\section{The Study}

The Emerging Infections Network (EIN) surveyed US infectious disease physicians to better assess the availability of $A$. fumigatus susceptibility testing in clinical settings, the frequency with which clinicians request susceptibility testing, and the degree to which clinicians have observed azole resistance. The EIN is a provider-based emerging infections sentinel network supported by the Centers for Disease Control and Prevention and sponsored by the Infectious Disease Society of America (12). During May-June 2016, EIN distributed surveys to 1,584 members by email and fax; 709 (45\%) responded.

Of the 709 respondents, nearly half $(348,49 \%)$ were familiar with the concept of azole-resistant $A$. fumigatus; $100(14 \%)$ were aware of the possible link to agricultural or environmental antifungal products. During the previous year, $364(51 \%)$ reported treating $\geq 1$ patient who had been diagnosed with aspergillosis. Of those, $136(38 \%)$ reported clinical failure of therapy for $\geq 1$ patient, despite $290(80 \%)$ physicians having used therapeutic drug monitoring to titrate azole therapy. Nine (2\%) treating physicians reported observing azole resistance in an azole-naive patient.

Overall, $224(62 \%)$ treating physicians who responded had access to susceptibility testing; 75 (21\%) lacked access; and an additional $65(18 \%)$ were unsure of availability. Of those with access, $182(81 \%)$ reported that testing was physician-prompted; $162(72 \%)$ reported that testing occurred off-site rather than in their hospital. For those reporting off-site testing, 8 (4\%) physicians typically received results within 1 week and 42 (19\%) reported receiving results $\geq 3$ weeks after request, excluding the minority $(n=6,4 \%)$, who were unsure. Of the 224 physicians who had access to susceptibility testing, 127 (57\%) reported that $\geq 1$ of their patients had an isolate tested, and $56(25 \%)$ reported that $>50 \%$ of patients had isolates tested. Forty-one $(19 \%)$ reported a patient isolate with resistance to $\geq 1$ azole, and $16(7 \%)$ reported a patient with a pan-azole-resistant isolate.

Sixteen $(8 \%)$ physicians practicing in the southern and $14(9 \%)$ practicing in the western US census regions reported seeing $>8$ patients who had aspergillosis during the previous year, compared with $5(3 \%)$ in the Northeast and $9(5 \%)$ in the Midwest $\left(\chi^{2}=6.3, p=0.18\right)$. Other findings were generally similar across regions, including proportions reporting clinical failure, azole resistance in azole-naive patients, susceptibility testing availability, routine versus physician-prompted testing, and location of testing.

Of 224 physicians with access to susceptibility testing, $8(16 \%)$ of 51 physicians in the South reported that $>50 \%$ of their patients' isolates were tested, compared with 17 (27\%) of 63 in the Midwest, 8 (21\%) of 37 in the Northeast, and 22 $(31 \%)$ of 70 from the West $\left(\chi^{2}=4.3, p=0.37\right)$. Of 51 physicians in the South, $13(26 \%)$ reported observing isolates resistant to $\geq 1$ azole, compared with 9 (14\%) of 63 from the Midwest, 5 (14\%) of 37 from the Northeast, and 13 (19\%) of 70 from the West $\left(\chi^{2}=10.2, p=0.04\right)$. Pan-azole-resistant isolates were reported by $4(8 \%)$ of 51 physicians in the South, $7(11 \%)$ of 63 in the Midwest, $2(5 \%)$ of 37 in the Northeast and $3(4 \%)$ of 70 in the West $\left(\chi^{2}=4.4, p=0.36\right)$.

In summary, approximately 50\% (348/709) of surveyed infectious disease physicians were familiar with azole-resistant $A$. fumigatus and 14\% (100/709) were aware of a possible link to environmental fungicide use. Of physicians who had treated patients diagnosed with aspergillosis within the past year, $21 \%(75 / 364)$ lacked access to susceptibility testing and $57 \%(127 / 224)$ who had access tested an isolate in the previous year. A small proportion of $19 \%$ $(41 / 224)$ reported observing any azole resistance and only $7 \%(16 / 224)$ reported pan-resistance. Of note, physicians in the southern states more commonly observed resistance to $\geq 1$ azole, compared with physicians from other regions.

Because only a small fraction of patients with invasive aspergillosis have a positive culture (13), a survey of resistance in culture-positive aspergillosis is not necessarily representative of all cases; but this fact highlights the importance of monitoring available cultures to inform broader practice. Another gap in our understanding of azole-resistant A. fumigatus is that the Clinical and Laboratory Standards Institute has not established breakpoints for azole susceptibility for $A$. fumigatus because inadequate clinical data exist 
to support breakpoints. The institute uses epidemiologic cutoff values, reflecting the minimal inhibitory concentration of $95 \%$ of wild-type isolates (13). However, there is some evidence that infection with resistant isolates by currently used thresholds is associated with worse outcomes in patients treated with azole monotherapy (13). Patients with hematologic or oncologic diseases are more likely to be infected with azole-resistant aspergillosis, and those with resistance have been shown to have higher case-fatality rates (2). However, it remains unclear to what degree these failures are attributable to underlying immunosuppression in these patients or to resistance-mediated treatment failure.

\section{Conclusions}

Our findings support that azole-resistant $A$. fumigatus infections, including those with pan-azole resistance, are occurring in the United States, and that broader susceptibility testing may be warranted to guide patient care. Systematic surveillance for aspergillosis, including collection of clinical data and isolates, could aid in detecting emergence of regional resistance patterns, assessing the role that resistance plays in treatment failure, and determining locally tailored treatment options. Awareness by physicians of azole-resistant aspergillosis and the possible link to environmental fungicide use are essential.

This work was funded by the Centers for Disease Control and Prevention (cooperative agreement no. 1 U50 CK000477-01).

Dr. Walker is an internal medicine physician who completed training in the Epidemic Intelligence Service in the Mycotic Diseases Branch, Division of Foodborne, Waterborne, and Environmental Diseases, National Center for Emerging and Zoonotic Infectious Diseases, CDC, Atlanta. She is currently investigating the incidence of influenza in adults with underlying conditions through the Southern Hemisphere Influenza and Vaccine Effectiveness Research \& Surveillance project supported by the Institute of Environmental Science and Research Limited), Wellington, New Zealand, in conjunction with CDC.

\section{References}

1. Verweij PE, Chowdhary A, Melchers WJG, Meis JF. Azole resistance in Aspergillus fumigatus: can we retain the clinical use of mold-active antifungal azoles? Clin Infect Dis. 2016;62:362-8. http://dx.doi.org/10.1093/cid/civ885

2. van der Linden JW, Snelders E, Kampinga GA, Rijnders BJ, Mattsson E, Debets-Ossenkopp YJ, et al. Clinical implications of azole resistance in Aspergillus fumigatus, The Netherlands, 2007-2009. Emerg Infect Dis. 2011;17:1846-54. http://dx.doi.org/ 10.3201/eid1710.110226

3. Wiederhold NP, Garcia Gil V, Lindner J, Sanders C, Fan H, Sutton A, et al. Evaluation of Cyp51A mechanisms of azole resistance in Aspergillus fumigatus isolates from the United States. Mycoses. 2015;58:55.

4. Patterson TF, Thompson GR III, Denning DW, Fishman JA, Hadley S, Herbrecht R, et al. Practice guidelines for the diagnosis and management of aspergillosis: 2016 Update by the Infectious Diseases Society of America. Clin Infect Dis. 2016;63:e1-60. http://dx.doi.org/10.1093/cid/ciw326

5. Mortensen KL, Mellado E, Lass-Flörl C, Rodriguez-Tudela JL, Johansen HK, Arendrup MC. Environmental study of azoleresistant Aspergillus fumigatus and other aspergilli in Austria, Denmark, and Spain. Antimicrob Agents Chemother. 2010;54:4545-9. http://dx.doi.org/10.1128/AAC.00692-10

6. Chowdhary A, Kathuria S, Xu J, Meis JF. Emergence of azole-resistant Aspergillus fumigatus strains due to agricultural azole use creates an increasing threat to human health. PLoS Pathog. 2013;9:e1003633. http://dx.doi.org/10.1371/ journal.ppat. 1003633

7. Verweij PE, Snelders E, Kema GH, Mellado E, Melchers WJ. Azole resistance in Aspergillus fumigatus: a side-effect of environmental fungicide use? Lancet Infect Dis. 2009;9:789-95. http://dx.doi.org/10.1016/S1473-3099(09)70265-8

8. Pham CD, Reiss E, Hagen F, Meis JF, Lockhart SR. Passive surveillance for azole-resistant Aspergillus fumigatus, United States, 2011-2013. Emerg Infect Dis. 2014;20:1498-503. http://dx.doi.org/10.3201/eid2009.140142

9. Wiederhold NP, Gil VG, Gutierrez F, Lindner JR, Albataineh MT, McCarthy DI, et al. First detection of TR34 L98H and TR46 Y121F T289A Cyp51 mutations in Aspergillus fumigatus isolates in the United States. J Clin Microbiol. 2016;54:168-71. http://dx.doi.org/ 10.1128/JCM.02478-15

10. van der Linden JW, Camps SM, Kampinga GA, Arends JP, Debets-Ossenkopp YJ, Haas PJ, et al. Aspergillosis due to voriconazole highly resistant Aspergillus fumigatus and recovery of genetically related resistant isolates from domiciles. Clin Infect Dis. 2013;57:513-20. http://dx.doi.org/10.1093/cid/cit320

11. Vazquez JA, Manavathu EK. Molecular characterization of a voriconazole-resistant, posaconazole-susceptible Aspergillus fumigatus isolate in a lung transplant recipient in the United States. Antimicrob Agents Chemother. 2015;60:1129-33. http://dx.doi.org/10.1128/AAC.01130-15

12. Pillai SK, Beekmann SE, Santibanez S, Polgreen PM. The Infectious Diseases Society of America Emerging Infections Network: bridging the gap between clinical infectious diseases and public health. Clin Infect Dis. 2014;58:991-6. http://dx.doi.org/ 10.1093/cid/cit932

13. Wiederhold NP, Patterson TF. Emergence of azole resistance in Aspergillus. Semin Respir Crit Care Med. 2015;36:673-80. http://dx.doi.org/10.1055/s-0035-1562894

Address for correspondence: Tiffany A. Walker, Centers for Disease Control and Prevention, 1600 Clifton Rd NE, Mailstop C09, Atlanta, GA 30329-4027, USA; email: twalker603@gmail.com 